OPEN Analyses of Interactions Between

SUBJECT AREAS:

PARASITE HOST

RESPONSE

PATHOGENS

PARASITE BIOLOGY

CLINICAL MICROBIOLOGY

Received

9 May 2013

Accepted

23 October 2013

Published

11 November 2013

Correspondence and requests for materials should be addressed to K.K. (kkato@obihiro. ac.jp) \section{Heparin and the Apical Surface Proteins of Plasmodium falciparum}

\author{
Kyousuke Kobayashi ${ }^{1,2,3}$, Ryo Takano ${ }^{4}$, Hitoshi Takemae ${ }^{1,4}$, Tatsuki Sugi i, , Akiko Ishiwa ${ }^{1,4}$, Haiyan Gong ${ }^{1}$,
} Frances C. Recuenco ${ }^{1,4}$, Tatsuya Iwanaga' ${ }^{1}$, Taisuke Horimoto' ${ }^{1}$ Hiroomi Akashi' \& Kentaro Kato ${ }^{1,4}$

'Department of Veterinary Microbiology, Graduate School of Agricultural and Life Sciences, the University of Tokyo, ${ }^{2}$ Division of Stem Cell Processing, Center for Stem Cell Biology and Regenerative Medicine, Institute of Medical Science, the University of Tokyo, ${ }^{3}$ Division of Host-Parasite Interaction, Department of Microbiology and Immunology, Institute of Medical Science, the University of Tokyo, ${ }^{4}$ National Research Center for Protozoan Disease, Obihiro University of Agriculture and Veterinary Medicine.

Heparin, a sulfated glycoconjugate, reportedly inhibits the blood-stage growth of the malaria parasite Plasmodium falciparum. Elucidation of the inhibitory mechanism is valuable for developing novel invasion-blocking treatments based on heparin. Merozoite surface protein 1 has been reported as a candidate target of heparin; however, to better understand the molecular mechanisms involved, we characterized the molecules that bind to heparin during merozoite invasion. Here, we show that heparin binds only at the apical tip of the merozoite surface and that multiple heparin-binding proteins localize preferentially in the apical organelles. To identify heparin-binding proteins, parasite proteins were fractionated by means of heparin affinity chromatography and subjected to immunoblot analysis with ligand-specific antibodies. All tested members of the Duffy and reticulocyte binding-like families bound to heparin with diverse affinities. These findings suggest that heparin masks the apical surface of merozoites and blocks interaction with the erythrocyte membrane after initial attachment.

M alaria is a major infectious disease worldwide; approximately 219 million people are infected with malaria annually, more than 660,000 of whom die (WHO report, 2012) ${ }^{1}$. This disease is caused by the infection of human erythrocytes with a protozoan parasite of the genus Plasmodium. Four humanspecific Plasmodium species are currently known, including P. falciparum, P. vivax, P. malariae, and P. ovale. Among these, P. falciparum causes the most virulent form of human malaria.

This parasite has two hosts: female mosquitoes of the genus Anopheles and humans. Sexual reproduction occurs in the mosquitoes, and intra-erythrocytic proliferation takes place in the human bloodstream via repeated cycles of erythrocyte invasion, cell division, and cell rupture. The process by which parasitic merozoites invade erythrocytes involves the following steps: attachment, apical reorientation, junction formation, and formation of a protective parasitophorous vacuole ${ }^{2,3}$.

Each invasion step is mediated by various proteins. In the initial attachment, merozoite surface proteins (MSPs) are thought to play an important role ${ }^{4}$. Apical membrane antigen-1 (AMA1) is believed to mediate active invasion by associating with rhoptry neck proteins (RONs) ${ }^{5,6}$. A number of factors are thought to mediate junction formation between merozoites and erythrocytes through sialic acid-dependent and -independent pathways; these factors include the Duffy binding-like (DBL) family that contains erythrocyte-binding antigen-175 (EBA-175), BAEBL (also known as EBA-140), JESEBL (also known as EBA-181), and EBL-1; and the reticulocyte binding-like (RBL) family, which contains $P$. falciparum reticulocyte-binding homolog 1 (PfRH1), PfRH2a, $\mathrm{PfRH} 2 \mathrm{~b}, \mathrm{PfRH} 4$, and PfRH5 ${ }^{7}$. These molecules recognize specific receptors on the erythrocyte surface, some of which have been identified, for example glycophorin A is a receptor for EBA-175, glycophorin B for EBL-1, glycophorin $\mathrm{C}$ for BAEBL, complement receptor 1 for PfRH4, and basigin for $\mathrm{PfRH}^{7}$. The function of the DBL and RBL proteins are redundant because knockout strains of all of the $d b l$ and $r b l$ genes except for PfRH5 have been generated and show insignificant loss of invasion efficiency?

Because the clinical manifestations of malaria are caused by asexual blood-stage parasites, a study of $P$. falciparum in this stage is important for developing effective treatments for malaria. In fact, almost all antimalarial drugs inhibit parasite growth through a blood stage-specific mechanism. However, parasites resistant to these drugs have emerged ${ }^{8,9}$ highlighting the need for novel drug targets. 
Recently, the inhibition of merozoite invasion by heparin was observed using real-time microscopy ${ }^{10}$. Merozoites reportedly attached to erythrocytes normally but could not proceed to the next step, apical reorientation, in the presence of heparin. Heparin is a polysaccharide consisting of repeating disaccharide units of an uronic acid molecule and a glucosamine molecule; it has abundant sulfate groups that confer its anionic charge. Several studies have reported the importance of these sulfate groups for the inhibitory activity of heparin ${ }^{10,11}$ and various sulfated polysaccharides, such as curdlan sulfate, dextran sulfate, pentosan sulfate, and fucoidan, $\lambda$ and 1 -carrageenans, as well as heparin and heparan sulfate (HS), inhibit the growth of blood-stage parasites in vitro ${ }^{11-15}$, although some sulfated polysaccharides, such as $\kappa$-carrageenan and chondroitin sulfates A (CSA) and C show no apparent inhibition ${ }^{11,14,15}$. Some sulfated polysaccharides including dextran sulfate ${ }^{16}$ and fucoidan ${ }^{17}$ have shown in vivo inhibitory activity in the blood-stage growth of $P$. berghei. In addition, artificially sulfated compounds, such as (poly)vinylsulfonate ${ }^{18}$ and sulfated cyclodextrin ${ }^{19}$, also reportedly inhibited growth in vitro and in vivo. These studies collective suggest that sulfated compounds have promise as novel antimalarial agents.

Although the inhibitory mechanism remains uncertain, our previous study showed that the addition of soluble heparin and HS inhibited the binding of BAEBL to erythrocytes by competitively inhibiting the binding to $H S$ on the erythrocyte surface ${ }^{20,21}$. However, this finding was not sufficient to explain the nearly complete invasion inhibition by heparin because BAEBL is not an essential ligand for the invasion ${ }^{22}$. Erythrocyte binding of some parasite ligands, including full-length MSP1, the C-terminal 42-kDa fragment of MSP1 (MSP1-42), and PfRH5 is disrupted by heparin ${ }^{10,11,23}$, raising the possibility that heparin-induced inhibition of the binding of multiple ligands results in invasion inhibition.

In this study, to better understand the mechanisms of invasion inhibition by sulfated polysaccharides, we examined the binding of heparin (as a model of a sulfated polysaccharide) to merozoite proteins or to the merozoite surface. Many molecules derived from parasite cultures, including erythrocyte-binding proteins, were found to interact with heparin. Further characterization demonstrated that the heparin-binding proteins are primarily localized in the apical organelles and are secreted to the apical tip. We found that proteins in the DBL and RBL families bound to heparin, and that some of them bound with high affinity. These results suggest that heparin-induced disruption of the interaction between the apical surface of merozoites and the erythrocyte surface contributes to invasion inhibition. This finding provides useful information for the development of novel antimalarial drugs and for understanding the role of HS on the erythrocyte surface during merozoite invasion.

\section{Results}

Heparin binding to the erythrocyte surface does not cause invasion inhibition of merozoite. Several previous studies have suggested that heparin or other anion saccharides inhibit the invasion of $P$. falciparum merozoites ${ }^{10,11,13,14,18,19,21,24}$, and some reports have suggested that the molecular targets of heparin are the merozoite proteins ${ }^{10,21,23}$. However, no report has excluded erythrocyte proteins as candidate targets. To investigate whether heparin has inhibitory effects on the susceptibility of erythrocytes to merozoites, we assessed merozoite invasion of erythrocytes that were preincubated with heparin (Fig. 1). When the erythrocytes were not washed, merozoites were unable to invade due to the presence of heparin. However, after washing, the susceptibility of the erythrocytes to merozoite invasion was restored to the same levels as in the control erythrocytes in the absence of heparin. These results suggest that heparin decreases the infectivity of merozoites, but not the susceptibility to erythrocytes.

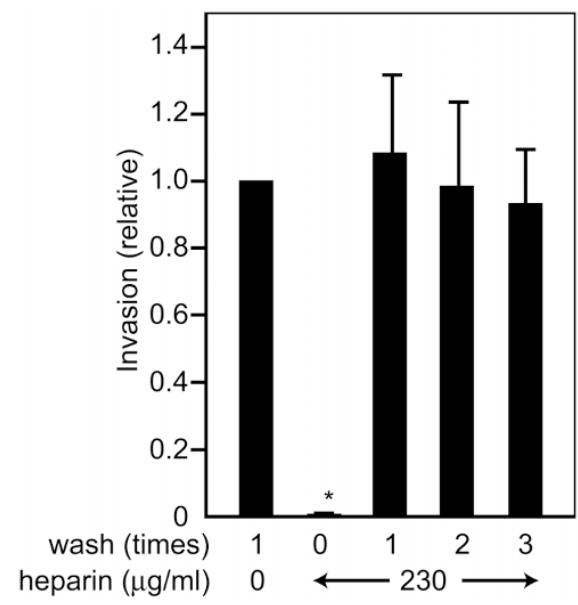

Figure $1 \mid$ The binding of heparin to the erythrocyte surface does not inhibit merozoite invasion. Three microliters of packed erythrocytes were mixed with $\mathrm{CM}$ in the absence or presence of $230 \mu \mathrm{g} / \mathrm{mL}$ heparin and incubated at $37^{\circ} \mathrm{C}$ overnight. The erythrocytes were washed $0-3$ times before being used for the invasion assays. The invasion rates were calculated by dividing the parasitemia of the test cultures by that of the cultures that lacked heparin. The results are shown as the means of three independent experiments, and the error bars represent standard errors. The asterisk $(*)$ indicates significant differences $(p<0.05)$ as determined by $t$-tests.

Heparin interacts with the merozoite surface. To investigate whether heparin interacts with the merozoite surface, we examined the binding between intact merozoites and heparin-agarose beads (Fig. 2). Several GFP- expressing merozoites stained with DAPI were observed on the surface of heparin-agarose beads (Fig. 2A). In the binding assay, the addition of soluble heparin decreased the punctate nuclear-staining pattern of the merozoites on the beads surface but not the diffuse pattern of background signals (Fig. 2B middle panel). On the other hand, the addition of soluble CSA, which has no or little inhibitory effect on merozoite invasion, caused no apparent decrease in merozoite binding to the bead surface (Fig. 2B right panel). This result demonstrates that the merozoite surface attaches to the heparin-agarose beads specifically and suggests that some heparinbinding proteins are expressed on the merozoite surface.

To prove that biotinylated heparin has the same binding properties as heparin, we demonstrated that biotinylated heparin correctly recognizes the surface of infected erythrocytes, as shown previously $^{25}$ (Fig. 3A). In addition, biotinylated heparin inhibited merozoite invasion at the same level as did unlabeled heparin (Fig. 3B). These results demonstrate that biotinylation has no effect on the nature of heparin. Isolated merozoites were incubated with biotinylated heparin and analyzed by means of flow cytometry (Fig. 3C). This assay detected the binding of biotinylated heparin to the merozoite surface. In addition, this binding was competitively inhibited by unlabeled heparin, indicating that biotinylated and unlabeled heparin recognize identical targets.

To detect the localization of heparin-binding proteins on the surface of merozoites, the merozoites treated with biotinylated heparin were observed by using confocal laser scanning microscopy (Fig. 3D). Heparin showed a similar localization pattern to that of EBA-175, demonstrating that heparin binds predominantly to the tip of the apical end.

Organelles containing abundant heparin-binding proteins were detected by observing biotinylated heparin bound to fixed and permeabilized merozoites on a glass slide (Fig. 3E). Compared with the localization of EBA-175 (microneme), MSP1-19 (surface), and the nucleus, heparin-binding proteins were found to be more abundant in the apical organelles, such as the micronemes and/or rhoptries. 

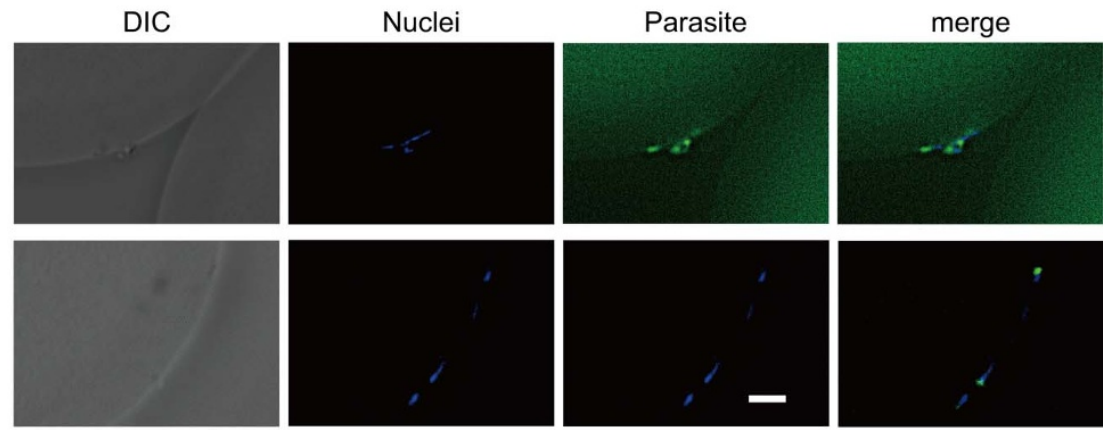

B
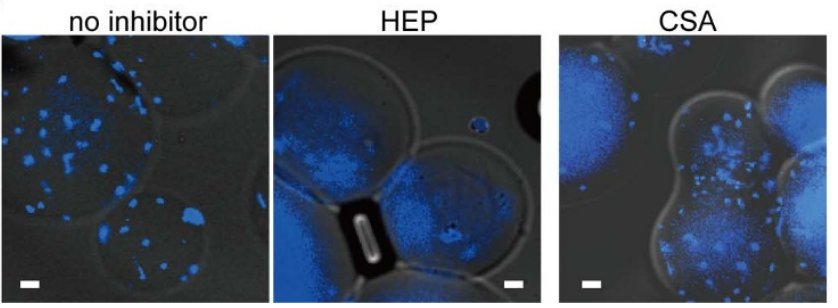

Figure $2 \mid$ Merozoites bind to heparin-agarose beads. (A) Heparin-agarose beads were incubated with a GFP-expressing parasite culture in the late schizont stage until the number of egressing merozoites increased. The beads separated from erythrocytes were washed, fixed, and stained with DAPI (nuclei). In this panel, differential interference contrast (DIC) images, fluorescent signals of the parasite nuclei and GFP, and the merged signals are shown. (B) During incubation of the beads with parasite cultures, $10 \mathrm{mg} / \mathrm{mL}$ soluble heparin (HEP), CSA, or PBS (no inhibitor) was added. After being collected, washed, and fixed, the beads were stained with TO-PRO-3. In panels A and B, the white bars represent $5 \mu \mathrm{m}$.

Many parasite proteins interact with heparin. To examine whether various proteins could bind to heparin, we incubated ${ }^{35} \mathrm{~S}$-labeled parasite proteins with a series of coated agarose beads and subjected them to autoradiography (Fig. 4A). Numerous parasite proteins bound to heparin-agarose beads, whereas no proteins bound to glutathione-Sepharose, Ni-NTA-agarose, or protein GSepharose beads.

Next, we evaluated the erythrocyte-binding potential of the parasite proteins adsorbed to the heparin-agarose beads. After adsorption to the beads, unbound proteins were subjected to an erythrocytebinding assay (Fig. 4B). The number of erythrocyte-binding proteins was drastically decreased by adsorption to heparin-agarose but not to glutathione-Sepharose beads. These results demonstrate that erythrocyte-binding proteins also bind to heparin.

Heparin-binding proteins can be separated from the schizont lysate by use of affinity chromatography with a heparin column. Taken together, the above findings suggest that heparin interacts with many erythrocyte-binding proteins secreted from the apical organelles to the apical surface. These characteristics closely resemble those of ligand molecules that mediate junction formation. Heparin has been reported to disrupt the erythrocyte binding of several ligand molecules involved in junction formation, including $\mathrm{BAEBL}^{21}$ and $\mathrm{PfRH}^{23}$. We, therefore, hypothesized that heparin might inhibit the binding of additional proteins involved in junction formation.

To isolate proteins that interact with heparin, we used affinity chromatography with a HiTrap heparin HP column (Fig. 5A). In this method, proteins weakly bound to heparin are eluted in buffer containing a lower concentration of $\mathrm{NaCl}$. The collected fractions are then analyzed by SDS-PAGE, followed by silver staining (Fig. 5B). In the E2-9 fractions, clear bands were detected, and the band patterns observed in each lane were reproducible.

The flow through (FT) and E3-9 fractions were analyzed by immunoblot analysis using specific antibodies against well-known ligands that mediate merozoite invasion. A full-length form of AMA1 (AMA1-83) was the most abundant in the FT but was detectable in E3-9 fractions, consistent with a previous report suggesting non-specific binding between heparin and $\mathrm{AMA1}{ }^{10}$. A $66-\mathrm{kDa}$ processed form of AMA1 (AMA1-66), however, did not bind to heparin and was detected only in the FT fraction, whereas the shorter forms of AMA1 (AMA1-52, AMA1-48, and AMA1-44) bound to heparin and were eluted in the E3-5 fractions. PfRON2 was also eluted in the E3-5 fractions. Although these two proteins and other RON members reportedly form a complex during invasion, it is not certain whether they bind to heparin as a complex.

The full-length and all processed forms of MSP1 containing the Cterminal region corresponding to MSP1-42 bound to heparin and were eluted in the E3-5 fractions. Therefore, the binding was relatively weak.

Among the DBL and RBL family members, BAEBL and PfRH1 showed the highest affinity for heparin (eluted in E9), whereas EBA175, JESEBL, and PfRH4 showed lower affinity (eluted in E3-5), as did MSP1. PfRH2 and PfRH5 (eluted in E6 and E7) showed greater affinity than did EBA-175, JESEBL, and PfRH4. These results indicate that all tested members of the DBL and RBL families bind heparin with different affinities. The ligands with relatively high affinity (BAEBL, PfRH1, PfRH2, and PfRH5) are associated with junction formation and localize to micronemes or rhoptries, consistent with the localization of the heparin-binding proteins shown in Figure 3.

Approximately $30 \%$ of the total parasite proteins, however, were detected in the bound and eluted fractions. Since it is unlikely that heparin-binding proteins constitute such a significant fraction of the total parasite proteins, we speculated that some parts of the eluted proteins bound to the column with relatively low specificity. To confirm the specificity, parasite proteins binding to the heparin affinity column were competitively eluted with increasing concentrations of soluble heparin. From $500 \mu \mathrm{g} / \mathrm{mL}$ to $10 \mathrm{mg} / \mathrm{mL}$, eluted proteins were detected by silver staining (Fig. 6A), suggesting that these proteins specifically recognized heparin. However, approximately $42 \%$ of the total proteins bound to the column were not eluted by up to $10 \mathrm{mg} / \mathrm{mL}$ heparin but were eluted by $2 \mathrm{M} \mathrm{NaCl}$ (Fig. 6B). These proteins theoretically contained two possible types: proteins that bound to the column nonspecifically and/or proteins that bound to heparin with too high an affinity to be eluted by $10 \mathrm{mg} / \mathrm{mL}$ heparin. 
A

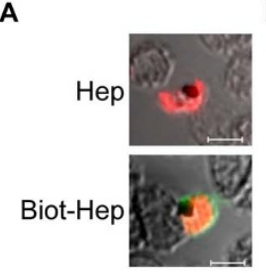

B

C
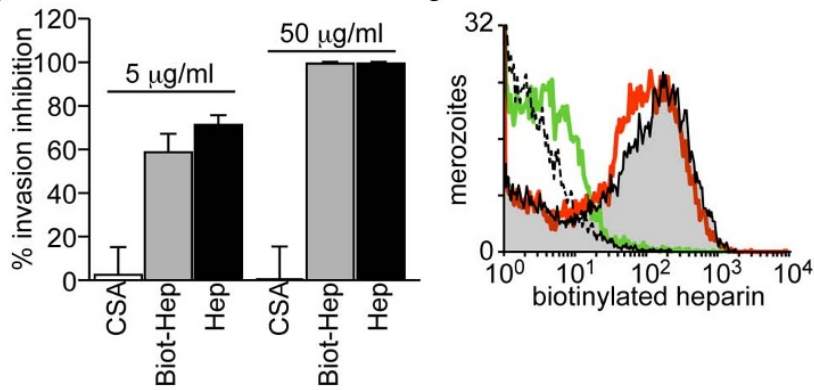

D

$\mathrm{E}$
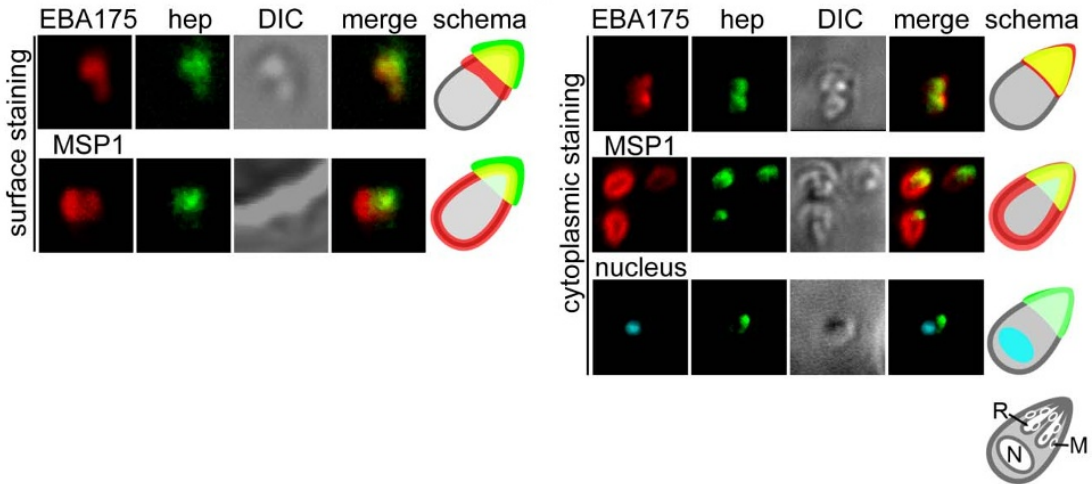

Figure 3 Heparin-binding proteins localize predominantly at the apical ends of merozoites. (A) The binding specificity of biotinylated heparin was verified. A parasite culture in the late schizont stage was smeared onto glass slides, fixed with methanol, and treated with a rabbit anti-EBA-175 antibody (red) and either unlabeled heparin (Hep) or biotinylated heparin (Biot-Hep; green). Signals were detected by using streptavidin-Alexa Fluor 488 and Alexa Fluor 633 secondary antibodies. The white bars represent $5 \mu \mathrm{m}$. (B) Invasion inhibitory activity of biotinylated heparin. Invasion inhibition assays were performed in the presence of 5 or $50 \mu \mathrm{g} / \mathrm{mL}$ CSA, biotinylated heparin (Biot-Hep), or unlabeled heparin (Hep). The percentages of invasion inhibition were calculated by dividing the parasitemia of the test cultures by that of the control cultures, multiplying the result by 100, and then subtracting the result from 100. The results are shown as the means of three independent experiments; the error bars represent standard errors. (C) Binding between heparin and merozoite surfaces. Parasite cultures at the late schizont stage were cultured with $50 \mu \mathrm{g} / \mathrm{mL}$ biotinylated heparin in the absence (grey) or presence of $1 \mathrm{mg} / \mathrm{mL}$ heparin (green line) or CSA (red line) for $1 \mathrm{~h}$. Free merozoites were isolated from the culture supernatant, stained with fluorescent streptavidin, and analyzed by flow cytometry. The dashed line shows untreated merozoites. (D) The heparin binding site on the merozoite surface. Biotinylated heparin was incubated with a parasite culture at the late schizont stage. When the number of egressing merozoites increased, the culture was fixed and stained with an antibody against EBA-175 (apical end) or MSP1-19 (surface). The biotinylated heparin and the antibody on the parasite surface were visualized by using fluorescent-labeled streptavidin or secondary antibodies. (E) Localization of heparin-binding proteins in merozoites. The localization of heparin-binding proteins was compared with EBA-175 (microneme and apical end), MSP1-19 (surface), or nuclei by immunofluorescent staining using biotinylated heparin, specific antibodies, and TO-PRO-3. (D), (E) The fluorescent signals of the marker proteins and heparin (hep), DIC images, merged fluorescent signals, and a schematic for their localization are shown. M, micronemes; R, rhoptries; N, nuclei.

The FT and fractions eluted with $500-10,000 \mu \mathrm{g} / \mathrm{mL}$ heparin and with $2 \mathrm{M} \mathrm{NaCl}$ were analyzed by immunoblotting using specific antibodies against MSP1 and the DBL and RBL proteins (Fig. 6C). All tested proteins were eluted by soluble heparin, suggesting that these proteins bind to heparin specifically. Two of these proteins, BAEBL and PfRH5, were eluted incompletely by $10 \mathrm{mg} / \mathrm{mL}$ heparin, suggesting that they have an extremely high affinity for heparin. The affinity determined by heparin elution should more faithfully represent the affinity to heparin than that determined by $\mathrm{NaCl}$ elution.

Heparin disaccharides (HDS) 1-S and CSA were used as control competitors, because inhibition of invasion by these compounds is much less efficiently than that by heparin ${ }^{10,11,14}$. In particular, because HDS 1-S were generated by digestion of heparin with heparinases and contain three major sulfate groups $(\mathrm{N}-, 2-\mathrm{O}-$, and 6-O-sulfate groups), the same concentration of heparin and HDS 1-S carry almost the same amount of negative charge. If only negative charge is important for binding to parasite proteins, HDS and/or CSA should also elute parasite proteins bound to the heparin-affinity column. However, few protein bands were detected in the fractions eluted by $1000-10000 \mu \mathrm{g} / \mathrm{mL}$ HDS, and no apparent bands were detected in the fractions eluted by CSA (Fig. 6A). A smaller proportion of proteins relative to the total proteins bound to the column were eluted by HDS (ca. 30\%) and CSA (ca. 25\%) compared with heparin (ca. 58\%) (Fig. 6B). The proteins eluted by HDS were assumed to bind to heparin only by electrostatic interaction or by specific recognition of a disaccharide unit. For these proteins, no parasite proteins associated with merozoite invasion were detected by immunoblotting (Fig. 6D).

Clone-specific disruption of the AMA1/RONs complex by heparin contributes little to the inhibition of invasion. It was recently revealed that the AMA1/RONs complex has a critical role in the merozoite invasion of $P$. falciparum ${ }^{5,6,26}$. Because we observed a relatively weak interaction between heparin and the complex components (AMA1 and RON2), we investigated the possibility that heparin inhibits AMA1/RONs complex formation.

As shown previously ${ }^{27}$, the bands corresponding to RON2, RON4, and RON5 coimmunoprecipitated with AMA1 (Fig. 7A). These bands from the 3D7 clone disappeared with the addition of the R1 peptide, whereas those from the HB3 clone did not. This R1 peptide reportedly binds to AMA1 and inhibits complex formation and merozoite invasion in a clone-specific manner; R1 peptide is effective for the $3 \mathrm{D} 7$ clone but not for the HB3 clone ${ }^{5,28}$. We confirmed this 
A

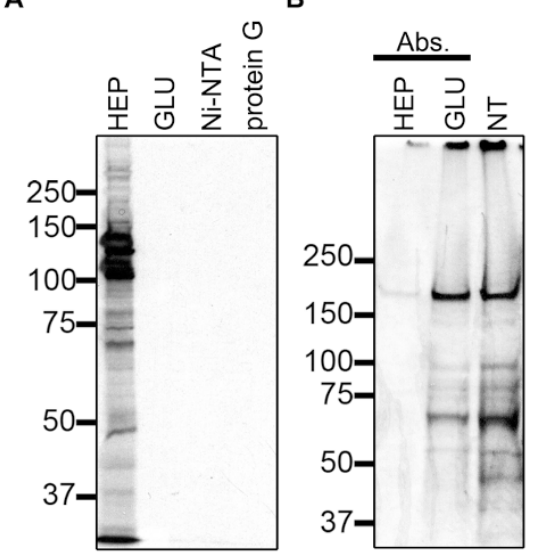

Figure $4 \mid$ Erythrocyte-binding proteins of parasites bind to heparinagarose beads. (A) Pull-down assays of radiolabeled culture supernatants of $P$. falciparum using heparin-agarose (HEP), glutathione-Sepharose (GLU), Ni-NTA-agarose, or protein G-Sepharose beads. (B) Erythrocytebinding assays of radiolabeled culture supernatants, which were preadsorbed to heparin-agarose (HEP) or glutathione-Sepharose (GLU) beads or were not pre-adsorbed (NT). Proteins that bound to the beads or erythrocytes were eluted and analyzed by means of autoradiography. The molecular masses $(\mathrm{kDa})$ are indicated on the left. specificity in our system and confirmed that the bands are RONs, consistent with previous reports.

Next, by using this assay, we examined whether heparin or CSA affects the complex formation (Fig. 7B). In the case of the HB3 clone, neither heparin nor CSA significantly decreased the coprecipitation of RON2 or RON4. Although RON5 of the HB3 clone appeared to be decreased by CSA (Fig. 7B), no such decrease occurred in any other experiments (data not shown). Densitometric analysis showed that formation of the complex between AMA1 and RONs was inhibited partially by the addition of either heparin or CSA (Fig. 7C), suggesting that, to induce the invasion inhibition, heparin targets molecular events other than those involving AMA1 and RONs. On the other hand, in the case of the 3D7 clone, AMA1/RONs complex formation was clearly inhibited by heparin but not by CSA. Densitometric analysis showed that the complex was partially disrupted by the addition of heparin but almost completely disrupted by the R1 peptide. These results demonstrate that heparin inhibits AMA1/RONs complex formation in a clone-specific manner.

If this heparin-induced complex disruption is a key factor for the invasion inhibition by heparin, heparin would be expected to cause no significant invasion inhibition of the HB3 clone. However, contrary to this expectation and without disruption of AMA1/RONs complex formation, heparin inhibited the invasion of the HB3 clone (Fig. 7D). Accordingly, disruption of this complex formation is not a true target of heparin.
A

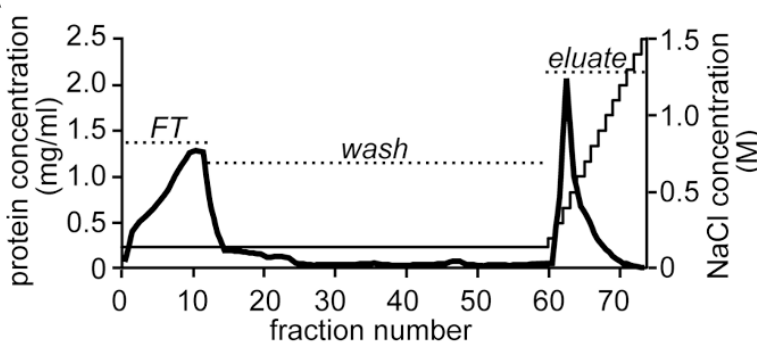

B

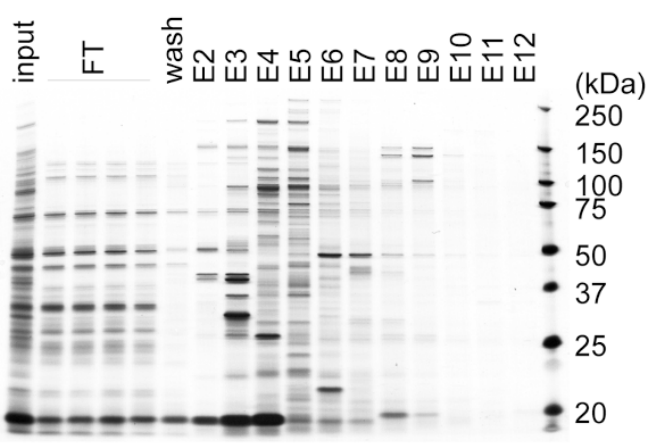

C

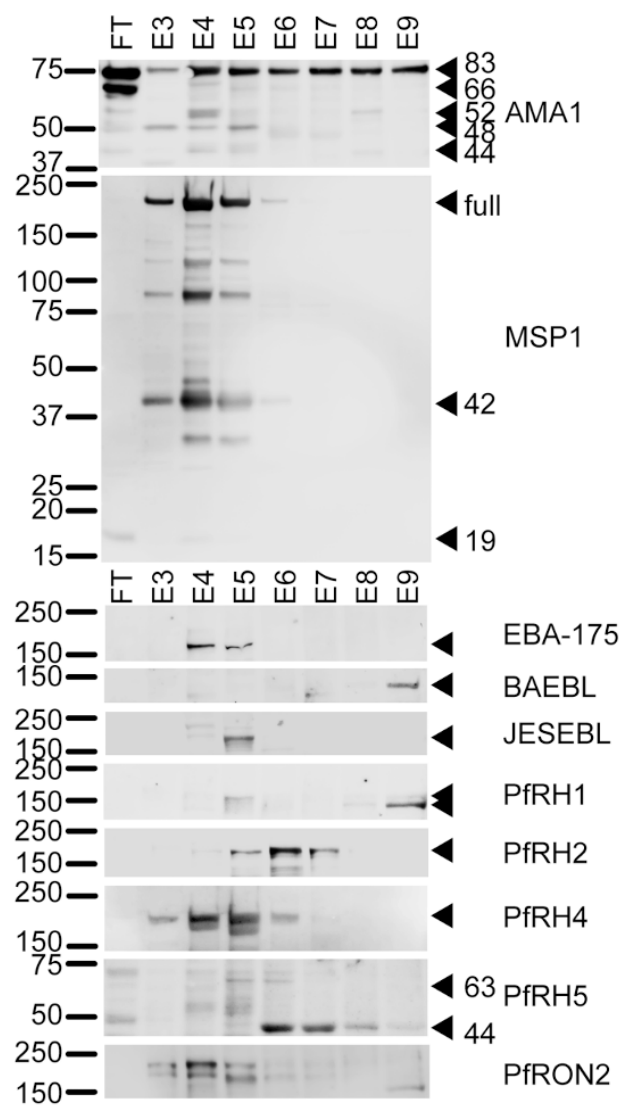

Figure $5 \mid$ Affinity chromatography of schizont proteins on a heparin column. (A) The elution profile of heparin-binding proteins of the $P$. falciparum HB3 clone. A schizont lysate was diluted with the binding buffer and separated by affinity chromatography on a heparin column. The proteins were washed and eluted from the column with a stepwise gradient of $\mathrm{NaCl}(0.2-1.5 \mathrm{M}$; thin line). Flow-through fractions of the lysate (FT), the wash buffer (wash), and the elution buffer (eluate) were collected (1.0 mL each) and subjected to protein quantification (thick line). (B) The eluate fractions containing proteins (E2-12) were analyzed by use of SDS-PAGE and silver staining. The molecular masses (kDa) are indicated on the right. (C) Eight fractions (FT and E3-9) were analyzed by immunoblotting. The arrowheads indicate specific bands. The molecular masses (kDa) are indicated on the left. 
A

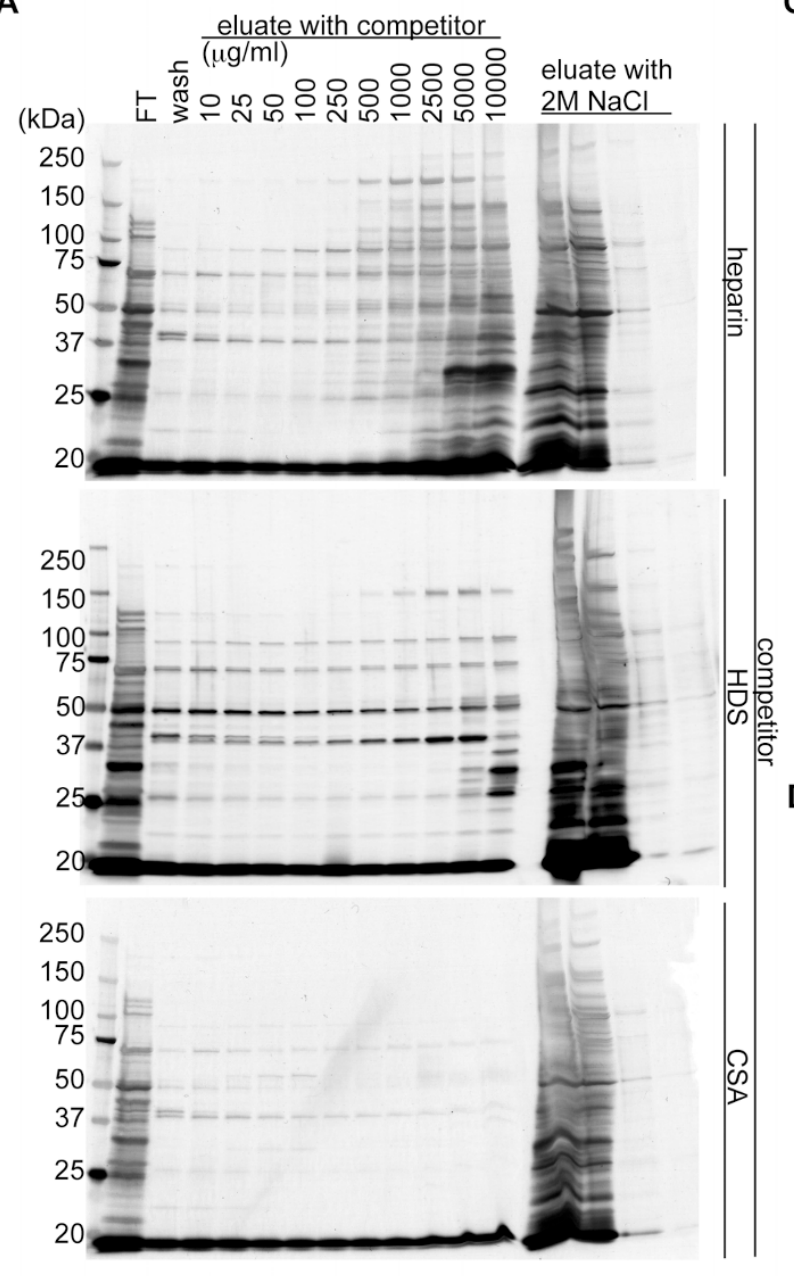

B

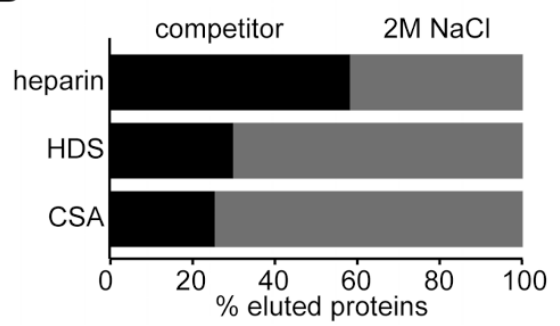

C
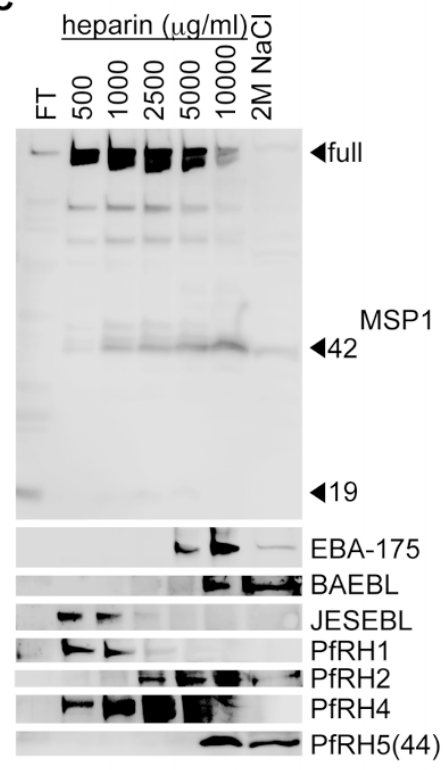

D

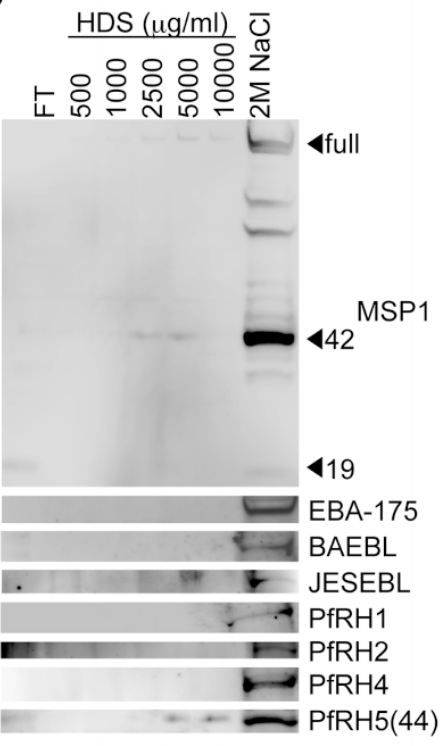

Figure 6 Elution of parasite proteins bound to the heparin column by soluble heparin or other sulfated compounds. (A) Parasite proteins eluted by soluble competitors (heparin, HDS, or CSA) were electrophoresed on SDS-PAGE and subjected to silver staining. Parasite proteins capable of binding to the heparin column were separated from a schizont lysate by using a HiTrap Heparin HP column with a bed volume of $1 \mathrm{~mL}$, washed with $10 \mathrm{~mL}$ of wash buffer, and eluted by $1 \mathrm{~mL}$ of increasing concentrations of the soluble competitors. After the competitive elution at the maximum concentration of $10 \mathrm{mg} / \mathrm{mL}$, the remaining proteins in the column were eluted with $2 \mathrm{M} \mathrm{NaCl}$. Flow-through fractions of the lysate (FT), proteins eluted by the wash buffer (wash), by the soluble competitors, or by $2 \mathrm{M} \mathrm{NaCl}$ were electrophoresed on SDS-PAGE and subjected to silver staining. The names of the competitors used for the elution are indicated on the right. The molecular masses $(\mathrm{kDa})$ are indicated on the left. (B) The protein amount eluted by the competitors or $\mathrm{NaCl}$ was estimated by quantitative densitometry of the silver-stained gel using Image J software (ver. $1.45 \mathrm{~s}$ ), because heparin in the eluted fractions interfered with the protein quantification by the Bradford method as used in Fig. 5 . The ratios of eluted proteins (by either the competitors or $2 \mathrm{M} \mathrm{NaCl)}$ to total proteins bound to the column was calculated. (C), (D) An unbound fraction (FT) and bound/eluted fractions with heparin (C) or HDS (D) were analyzed by immunoblotting.

\section{Discussion}

To reveal the mechanisms by which heparin inhibits merozoite invasion, it is important to understand when the inhibition occurs and whether this inhibition results from heparin interaction with the host cells or with the parasites. Previous studies found that heparin had little to no effect on the intracellular development of schizont- or ring-stage parasites ${ }^{10,11,24}$. Instead, direct evidence using real-time microscopy suggested that heparin inhibits merozoite invasion ${ }^{10}$; however, it remained clear whether heparin targets merozoites or erythrocytes for invasion inhibition. This study showed that heparin has an insignificant effect on erythrocytes with regard to merozoite invasion. Therefore, future studies should focus on interactions between merozoites and heparin to reveal the inhibitory mechanisms of heparin.

We found that various parasite proteins bind to heparin, by using pull-down assays of ${ }^{35} \mathrm{~S}$-labeled parasite proteins and heparin affinity 
A

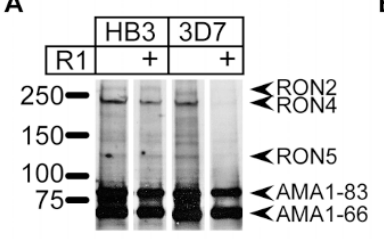

C

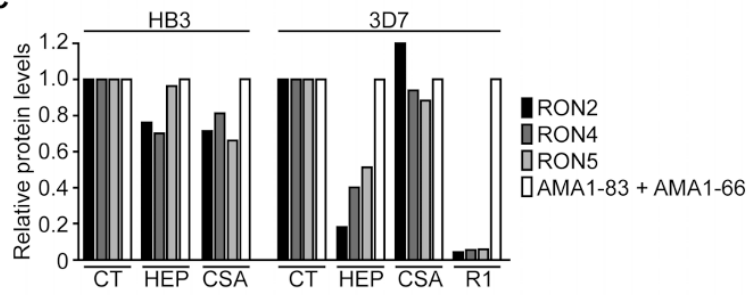

D

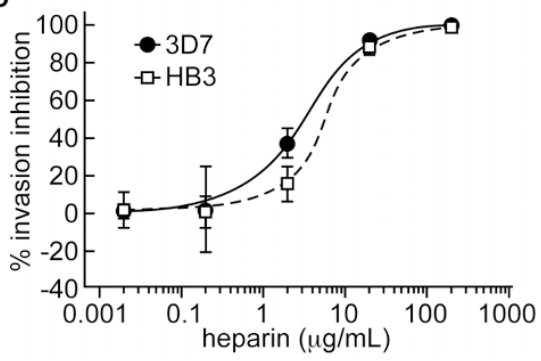

Figure 7 | Clone-specific disruption of the AMA1/RONs complex by heparin contributes little to invasion inhibition. (A), (B) Radiolabeled schizont lysates from the $\mathrm{HB} 3$ or 3D7 clones were immunoprecipitated with the 28G2 monoclonal antibody (anti-AMA1) in the presence or absence of $1 \mathrm{mg} / \mathrm{mL}$ heparin (HEP), CSA, or the R1 peptide, and then subjected to autoradiographic analysis. The arrowheads indicate the bands that correspond to each molecule. The bands corresponding to RON2 from the HB3 clone exhibited slower mobility on SDS-PAGE than did those from the 3D7 clone. The asterisk in panel B denotes a nonspecific band that did not appear consistently but did not disappear in the presence of the $\mathrm{R} 1$ peptide. The molecular masses $(\mathrm{kDa})$ are indicated on the left. (C) Relative protein levels of each RON protein co-immunoprecipitated with AMA-1 in the absence or presence of heparin, CSA, or the R1 peptide. The optical densities of each band were measured with Image J software. Data are shown as the means of two independent experiments. (D) Invasion inhibitory activities of heparin against the P. falciparum $\mathrm{HB} 3$ and 3D7 clones. Invasion assays were performed in the presence of heparin at a final concentration of $0.02,0.2,2,20$, or $200 \mu \mathrm{g} / \mathrm{mL}$. The percentages of invasion inhibition were calculated by dividing the parasitemia of the test cultures by that of the control cultures, multiplying the result by 100 , and then subtracting the result from 100. The results are shown as the means of three independent experiments; the error bars represent standard deviations.

chromatography of mature schizont proteins. Heparin is reportedly capable of binding to various mammalian proteins: coagulation factors, lipoprotein lipases, growth factors, and DNA- and RNA-associated enzymes ${ }^{29,30}$. Although heparin may bind to various types of parasite proteins, as it does in mammals, we found that almost all of the erythrocyte-binding proteins of $P$. falciparum have the capacity to bind to heparin. This finding raises the possibility that the binding of heparin to various erythrocyte-binding proteins leads to their binding inhibition, resulting in invasion inhibition.

Our study also showed direct binding between merozoites and heparin-agarose beads, which strongly suggests that heparin binds directly to the merozoite surface. In addition, immunofluorescence studies demonstrated that the heparin localization was restricted to the apical surface. The intracellular localization of heparin-binding proteins was observed mainly in apical organelles, such as micronemes or rhoptries.

Curiously, although this study and a previous one ${ }^{10}$ showed that heparin could bind to MSP1-42, which is localized on the merozoite surface, we did not find co-localization of MSP1 with heparin on the merozoite surface. We speculate that because MSP1-42 forms complexes with other MSP fragments ${ }^{4}$, the heparin-binding sites in MSP1-42 may have been masked and inaccessible. In addition, because of its relatively low affinity for MSP1-42, heparin preferentially binds to proteins with higher affinities that are localized on the apical surface of merozoites.

Our data suggest that heparin-binding proteins include the erythrocyte-binding proteins that are localized in the apical organelles and that they are transported to the apical surface. These characteristics are similar to those of the ligand molecules that are associated with junction formation. However, junction formation is mediated by multiple receptor-ligand interactions. To disrupt junction formation, heparin would have to block either the essential interaction or multiple alternative interactions. The former possibility was examined. Formation of the AMA1/RONs complex, one of the few essential types of machinery needed for junction formation, was disrupted by heparin in the 3D7 clone but not in the HB3 clone. However, despite no apparent disruption of the complex, heparin sufficiently blocked the invasion of the HB3 clone, suggesting that complex disruption is not a main target of heparin. In addition, when comparing the two live video microscopic studies of merozoite invasion in the presence of the R1 peptide or heparin, we see that the R1 peptide did not interfere with primary attachment or reorientation but participated in further processes despite the appearance of echinocytosis ${ }^{31}$, whereas heparin interfered with reorientation but not with primary attachment ${ }^{10}$. Therefore, these two inhibitors inhibit different invasion processes, with heparin inhibiting molecular events other than AMA1/RONs complex formation.

Our findings support the latter possibility that multiple alternative interactions are involved, because all of the DBL and RBL family members that we tested bound to heparin. A remarkable finding is that heparin bound to PfRH2, PfRH4, and PfRH5, all ligands that mediate sialic acid-independent pathways ${ }^{7}$. This finding suggests that heparin inhibits both sialic acid-dependent and sialic acid-independent pathways. This possibility is consistent with a previous finding that both sialic acid-dependent and-independent invasion of $P$. falciparum merozoites is inhibited to the same degree by heparin ${ }^{10}$.

Do all of these interactions really occur on the merozoite surface? To answer this question, we must look at when the different parasite ligands bind to heparin. According to the model proposed by Singh et $a .^{32}$, the DBL proteins localized in micronemes, but not the RBL proteins localized in rhoptries, are secreted onto the merozoite surface during merozoite egress and subsequently interact with their specific receptors during the invasion. These interactions trigger the secretion of rhoptry proteins to the apical surface. Therefore, the DBL family proteins are accessible to heparin before initial attachment, but the RBL family proteins would be inaccessible. Binding of heparin to the DBL family proteins would then to lead to a blockade of the receptor-ligand interactions, resulting in a failure of the RBL-family proteins to secrete to the surface. Alternatively, the binding of heparin to the DBL family proteins may trigger the secretion of rhoptry proteins before junction formation. However, there is contradictory evidence for PfRH4 and EBA-175 playing equivalent roles in merozoite invasion ${ }^{33,34}$. Further detailed research on the molecular events that surround merozoite invasion is required to fully understand the true targets of heparin.

Our model is consistent with a previous video microscopic observation $^{10}$. According to this previous report, in the presence of heparin, merozoites attach to the erythrocyte surface normally but do not proceed to the next step: deformation of the erythrocyte membrane and/or apical reorientation of the merozoites. We found that 
heparin binding was restricted at the apical surface of merozoites, implying that other regions of the merozoite surface normally attach to the erythrocyte surface, a finding that is consistent with the result of the video microscopy. Under normal conditions, this attachment triggers deformation of the erythrocyte membrane at the site of attachment, which is predicted to be the actual mechanism of apical reorientation ${ }^{35,36}$. Yet, in the presence of heparin, membrane deformation is inhibited ${ }^{10}$. Although neither the parasite nor the erythrocyte factors involved in this process have been identified, heparin must interfere with such factors to inhibit membrane deformation. However, some studies have suggested that erythrocyte deformation is not an essential step for merozoite inva$\operatorname{sion}^{31,35}$. Given that heparin inhibits merozoite invasion almost completely, it must inhibit essential steps. In our model, the binding of heparin to the apically expressed parasite ligands inhibits junction formation between the apical surface of merozoites and the erythrocyte membrane, which is essential for merozoite invasion. Consistent with this model, in the movie data of Boyle et al. ${ }^{10}$, after attachment to the erythrocyte surface, the merozoites appeared to move and roll on the erythrocyte surface in the presence of heparin, but do not form tight junction. Therefore, our model also fit with these previous observations.

Heparin inhibits the invasion of Toxoplasma gondii, which, like $P$. falciparum, belongs to the phylum Apicomplexa. However, a much greater concentration of heparin $\left(\mathrm{IC}_{50}>1.0 \mathrm{mg} / \mathrm{mL}\right)$ is required for invasion inhibition of $T$. gondii, compared with that needed for $P$. falciparum $\left(\mathrm{IC}_{50}=5-10 \mu \mathrm{g} / \mathrm{mL}\right.$ ). Moreover, CSA also inhibits the invasion of $T$. gondii at comparable levels to heparin ${ }^{37}$. Given the numerous similarities among host cell invasion mechanisms, the reason for these differences between $T$. gondii and P. falciparum is unclear. We assume that the differences are due to the repertoires of ligand molecules used for the parasite invasion. The DBL and RBL families, which were shown to bind to heparin in this study, are specific to Plasmodium species. If heparin is primarily targeted to these family members, it should inhibit the host cell invasion of $P$. falciparum merozoites more effectively than that of $T$. gondii tachyzoites.

Several studies have reported that some sulfated polysaccharides inhibit the growth of blood-stage Plasmodium parasites in vitro and in vivo ${ }^{10-13,15-17,21}$. Some compounds to which sulfate groups were artificially added have been reported to have inhibitory effects on parasite growth in vitro and in vivo ${ }^{18,19}$. These data suggest that the sulfate groups on these polysaccharides and these compounds play an important role in growth inhibition, and may have potential as a new type of antimalarial drugs. Here, we propose a model for the inhibition mechanism by heparin, a representative of the sulfated polysaccharides. However, the question remains: are the structural features or negative charge of sulfated compounds important? In this regard, our data suggest that disaccharide heparin fails to bind to the DBL and RBL proteins, in agreement with previous similar reports ${ }^{10,11}$. Therefore, a polysaccharide structure consisting of at least 5 monosaccharide units with sulfate groups is required for the invasion inhibition.

In summary, here, we show that heparin targets multiple molecules that mediate the parasite invasion of erythrocytes, providing important clues for the development of antimalarial drugs to block the merozoite. Because multiple molecules are not likely to simultaneously acquire resistance to heparin, the risk for emergence of parasites resistant to heparin is assumed to be extremely low. In agreement with this inference, a previous study indicated that no parasite with increased resistance to heparin was selected in the presence of heparin ${ }^{10}$. By identifying the target molecules of heparin, it may be possible to develop compounds that bind those target even more specifically than heparin. Therefore, the result of this study could help in the development of new therapies against malaria.

\section{Methods}

P. falciparum cultures. The P. falciparum clones HB3, 3D7, and GFP-expressing parasite (3D7HT-GFP) were obtained from the Malaria Research and Reference Reagent Resource Center (MR4; American Type Culture Collection, Manassas, VA). $\mathrm{B}+$ erythrocytes from a single individual were used in all cultures and experiments. The cells were washed twice in incomplete RPMI-1640 medium containing $25 \mathrm{mM}$ HEPES and $367 \mu \mathrm{M}$ hypoxanthine, and then stored at $50 \%$ hematocrit and $4{ }^{\circ} \mathrm{C}$. The cultures were maintained at $1 \%-3 \%$ hematocrit in complete medium (CM) that was composed of incomplete medium containing $5 \mathrm{mg} / \mathrm{mL}$ AlbuMAX II (Invitrogen, Carlsbad, CA), $27 \mathrm{mM} \mathrm{NaHCO}$, and $10 \mu \mathrm{g} / \mathrm{mL}$ gentamicin as previously described $^{38}$.

Antibodies. The anti-EBA-175 antibody was produced by immunization of rabbits with the synthetic peptides CYKVNEREDERTLTKE and CMNRESDDGELYDEN which comprise amino acids 1092-1107 and 1117-1130, respectively, of EBA-175 (GenBank Accession No. XP_001349207). These regions overlap the previously reported region ${ }^{39}$. A rabbit was immunized as previously described ${ }^{21}$. The rabbit polyclonal antibody against the C-terminal 19-kDa fragment of MSP1 (MSP1-19) and a rat monoclonal antibody against AMA1 $(28 \mathrm{G} 2)^{40}$ were obtained from MR4. The rabbit anti-AMA1-C1 antibody was kindly provided by Drs. Carol A. Long and. Kazutoyo Miura (National Institute of Health, Bethesda, MD, USA) ${ }^{41}$. The rat antiJESEBL antibody and the rabbit anti-PfRH4 antibody were kindly provided by Dr. Louis H. Miller (National Institute of Health) ${ }^{42,43}$. The rabbit anti-clag3.1 antibody was kindly provided by Dr. Osamu Kaneko (Nagasaki University, Japan) ${ }^{44}$. The rabbit antibody against PfRON2 was kindly provided by Dr. Takafumi Tsuboi (Ehime University, Japan $)^{26}$. The mouse anti-PfRH1, PfRH2a/b, and PfRH5 antibodies were obtained by immunization of mice with recombinant proteins or synthetic peptides, identical to those previously reported: $\mathrm{R} 515$ for $\mathrm{PfRH}^{45}, \mathrm{~S} 3$ for $\mathrm{PfRH} 2 \mathrm{a} / \mathrm{b}^{46}$, and the peptide LIKCIKNHENDFNKIC for PfRH ${ }^{47}$.

Polysaccharides. Heparin, CSA, and biotinylated heparin were purchased from Sigma-Aldrich (St. Louis, MO) and heparin disaccharide 1-S from Dextra Laboratory (Reading, UK). The R1 peptide VFAEFLPLFSKFGSRMHILK was synthesized (Operon Biotechnologies, Tokyo, Japan).

Invasion inhibition assays. Invasion assays were performed as described previously ${ }^{48}$. Briefly, $3 \mu \mathrm{L}$ of packed erythrocytes were mixed with $6 \times 10^{5}$ schizonts, which were enriched by the Percoll-sorbitol method $25 \mathrm{~h}$ after synchronization with the $5 \% \mathrm{D}$-sorbitol method. After being cultured for $20 \mathrm{~h}$, parasitemias of ring-stage parasites were evaluated by using Giemsa-stained culture smears.

Immunofluorescent staining. To detect the binding of merozoites to heparinagarose beads, purified schizont-stage parasites were suspended in CM. The suspension was then mixed with heparin-agarose beads and $10 \mathrm{mg} / \mathrm{mL}$ soluble heparin or CSA and incubated at $37^{\circ} \mathrm{C}, 5 \% \mathrm{CO}_{2}$, and $5 \% \mathrm{O}_{2}$. Parallel cultures were prepared by the addition of fresh erythrocytes to the purified schizonts and monitored for increases in ring forms to determine the optimal time to observe parasite egress. When the number of egressing parasites was estimated to be increasing in the culture, the beads were suspended in $10 \mathrm{~mL}$ of ice-cold ICM and centrifuged at $120 \times g$ for $10 \mathrm{sec}$ at $4^{\circ} \mathrm{C}$ to precipitate only the beads. The supernatant containing parasitized erythrocytes and free merozoites was removed. The beads were then washed with ICM three times with centrifugation under the same condition, fixed, and blocked as described previously ${ }^{49}$. After incubation with $5 \mu \mathrm{M}$ DAPI or $2 \mu \mathrm{M}$ TO-PRO-3 iodide (Invitrogen) for $1 \mathrm{~h}$ at room temperature, the beads were rinsed three times with PBS. Lastly, 3-4 $\mu \mathrm{L}$ of the suspension was spotted onto a glass slide, mounted under a coverslip, and observed under a fluorescent microscope (model BZ-9000; Keyence, Osaka, Jaman) and/or a confocal laser-scanning microscope (LSM510; Carl Zeiss, Oberkochen, Germany).

To detect heparin binding sites on the merozoite surface, a synchronous culture at the late schizont stage was mixed with $30 \mu \mathrm{g} / \mathrm{mL}$ biotinylated heparin and cultured. Parallel cultures were monitored to determine the optimal time to observe parasite egress. When the number of egressing parasites was estimated to be increasing in the cultures, the cultures were centrifuged at $800 \times g$ for $5 \mathrm{~min}$ at room temperature and washed with ICM once and with PBS twice. The cells were then fixed with phosphate buffer containing $1 \%$ paraformaldehyde and $0.1 \%$ glutaraldehyde for 20 min on ice and subsequently washed with PBS. After being blocked with $1 \%$ bovine serum albumin (BSA) overnight at $4^{\circ} \mathrm{C}$, they were incubated with an antibody for $30 \mathrm{~min}$ at $37^{\circ} \mathrm{C}$. After being washed, the slides were incubated with $2 \mu \mathrm{g} / \mathrm{mL}$ Alexa Fluor 633 goat anti-rabbit IgG $(\mathrm{H}+\mathrm{L}$ ) (Invitrogen) and $5 \mu \mathrm{g} / \mathrm{mL}$ streptavidin-Alexa Fluor 488 (Invitrogen). After being washed, the slides were observed as described above.

To detect the localization of heparin-binding molecules in merozoites, a synchronous culture at the late schizont stage was smeared and fixed with methanol for $5 \mathrm{~min}$ at room temperature. After being blocked with $0.3 \%$ BSA for $30 \mathrm{~min}$ at $37^{\circ} \mathrm{C}$, the slides were incubated with $50 \mu \mathrm{g} / \mathrm{mL}$ biotinylated heparin and an antibody for $30 \mathrm{~min}$ at $37^{\circ} \mathrm{C}$. After being washed, the slides were incubated with secondary antibodies and observed as described above.

Metabolic labeling of parasite proteins. Metabolic labeling of parasite proteins with $\mathrm{L}-\left[{ }^{35} \mathrm{~S}\right]$ methionine and L- $\left[{ }^{35} \mathrm{~S}\right]$ cysteine was performed as previously described ${ }^{50}$. 
Pull-down assays. Pull-down assays were performed as previously described ${ }^{21}$ using heparin-agarose, Ni-NTA-agarose, glutathione-Sepharose (GE Healthcare, Buckinghamshire, UK), or protein G-Sepharose beads (GE Healthcare).

Erythrocyte-binding assays. Erythrocyte-binding assays were performed as previously described ${ }^{50}$.

Affinity chromatography using a heparin column. To collect mature schizonts containing merozoites, the egress of schizonts was halted by adding E-64 cysteine protease inhibitor (Sigma-Aldrich) to a highly synchronized culture of $P$. falciparum HB3 clone at the schizont stage $\mathrm{e}^{51}$. After 3-8 h, parasite lysates were prepared by saponin treatment ${ }^{52}$ and were solubilized with lysis buffer $\left[10 \mathrm{mM} \mathrm{NaH}_{2} \mathrm{PO}_{4}(\mathrm{pH}\right.$ 6.7) containing $100 \mathrm{mM} \mathrm{NaCl}, 1 \mathrm{mM}$ EDTA, and 1.0\% Triton X-100] and a protease inhibitor cocktail (Roche Diagnostics, Mannheim, Germany) for $1 \mathrm{~h}$ at $4{ }^{\circ} \mathrm{C}$. After centrifugation at $91,000 \times g$ for $1 \mathrm{~h}$ at $4^{\circ} \mathrm{C}$, the supernatant was diluted with binding buffer [ $10 \mathrm{mM} \mathrm{NaH}_{2} \mathrm{PO}_{4}$ (pH 6.7) containing $100 \mathrm{mM} \mathrm{NaCl}$ ] and applied to a HiTrap heparin HP column (GE Healthcare) that was equilibrated with binding buffer. The column was washed with wash buffer $\left[10 \mathrm{mM} \mathrm{NaH} \mathrm{PO}_{4}(\mathrm{pH} 6.7)\right.$ containing $100 \mathrm{mM} \mathrm{NaCl}$ and $0.1 \%$ Triton $\mathrm{X}-100$ ] and proteins were eluted from the column with a stepwise gradient of $0-1.5 \mathrm{M} \mathrm{NaCl}$ or $0-10 \mathrm{mg} / \mathrm{mL}$ soluble heparin or CSA in wash buffer. Protein concentrations in each fraction $(1.0 \mathrm{~mL})$ were analyzed by using the Protein Quantification Kit-Raid (Dojindo, Kumamoto, Japan).

Immunoblot analysis. Immunoblot analysis was performed as previously described $^{53}$.

Immunoprecipitation. Immunoprecipitation was performed as previously described ${ }^{50}$. Briefly, synchronized and purified schizonts were labeled with L$\left[{ }^{35} \mathrm{~S}\right]$ methionine and L- $\left[{ }^{35} \mathrm{~S}\right]$ cysteine for $3 \mathrm{~h}$. The parasites were harvested by centrifugation and washed once with PBS. Proteins were extracted from the parasite pellet in Lysis buffer containing one of the inhibitors. After centrifugation, the supernatants were preincubated at $4{ }^{\circ} \mathrm{C}$ for $1 \mathrm{~h}$ with an equal volume of protein GSepharose beads. Aliquots of the recovered supernatants were diluted with IP buffer [10 $\mathrm{mM} \mathrm{NaH}_{2} \mathrm{PO}_{4}$ (pH 6.7) containing $100 \mathrm{mM} \mathrm{NaCl}, 0.5 \%$ Triton X-100, and $0.5 \%$ BSA] containing one of the inhibitors, incubated with an antibody for $2 \mathrm{~h}$, then incubated for $1.5 \mathrm{~h}$ at $4{ }^{\circ} \mathrm{C}$ with $50 \%$ protein G-Sepharose beads. After this incubation, the beads were washed twice with NETT buffer [ $50 \mathrm{mM}$ Tris- $\mathrm{HCl}$ ( $\mathrm{pH} 7.4), 0.15 \mathrm{M}$ $\mathrm{NaCl}, 5 \mathrm{mM}$ EDTA, and $0.5 \%$ Triton X-100] containing $0.5 \% \mathrm{BSA}$, and three times with NETT. Finally, proteins were eluted from the beads with sodium dodecyl sulfatepolyacrylamide gel electrophoresis (SDS-PAGE) sample buffer and analyzed by autoradiography.

Flow cytometry. Synchronous cultures at the late schizont stage with increasing numbers of ring forms were mixed with $50 \mu \mathrm{g} / \mathrm{mL}$ biotinylated heparin and cultured for $1 \mathrm{~h}$. The cultures were centrifuged at $500 \times g$ for $5 \mathrm{~min}$ at room temperature to separate free merozoites from erythrocytes. The supernatants containing free merozoites were passed through 1.2 - $\mu \mathrm{m}$ filters (Whatman, UK) and centrifuged at $3300 \times g$ for $5 \mathrm{~min}$ at room temperature. The merozoites were then washed with FACS buffer consisting of PBS containing $2 \%$ fetal calf serum and $0.1 \%$ sodium azide and were incubated with $10 \mu \mathrm{g} / \mathrm{mL}$ ethidium bromide and $10 \mu \mathrm{g} / \mathrm{mL}$ streptavidinAlexa Fluor 488 on ice for $1 \mathrm{~h}$. After being washed three times with FACS buffer, the merozoites were analyzed on a FACSCalibur system using Cell Quest software (Becton Dickinson, San Jose, CA). To distinguish merozoites from erythrocytes or cell debris, merozoites were gated by size in the forward scatter channel and by fluorescence in FL2.

1. World Health Organization. World malaria report: $2012<$ www.who.int/ malaria $>$ (2012)

2. Aikawa, M., Miller, L. H., Johnson, J. \& Rabbege, J. Erythrocyte entry by malarial parasites. A moving junction between erythrocyte and parasite. J. Cell Biol. 77, 72-82 (1978).

3. Dvorak, J. A., Miller, L. H., Whitehouse, W. C. \& Shiroishi, T. Invasion of erythrocytes by malaria merozoites. Science 187, 748-750 (1975).

4. Holder, A. A. The carboxy-terminus of merozoite surface protein 1: structure, specific antibodies and immunity to malaria. Parasitology 136, 1445-1456 (2009).

5. Richard, D. et al. Interaction between Plasmodium falciparum apical membrane antigen 1 and the rhoptry neck protein complex defines a key step in the erythrocyte invasion process of malaria parasites. J. Biol. Chem. 285, 14815-14822 (2010).

6. Srinivasan, P. et al. Binding of Plasmodium merozoite proteins RON2 and AMA1 triggers commitment to invasion. Proc. Natl. Acad. Sci. U. S. A. 108, 13275-13280 (2011).

7. Tham, W. H., Healer, J. \& Cowman, A. F. Erythrocyte and reticulocyte bindinglike proteins of Plasmodium falciparum. Trends Parasitol 28, 23-30 doi:10.1016/ j.pt.2011.10.002 (2012)

8. Dondorp, A. M. et al. Artemisinin resistance in Plasmodium falciparum malaria. N. Engl. J. Med. 361, 455-467 doi:10.1056/NEJMoa0808859 (2009).

9. Borrmann, S. et al. Declining responsiveness of Plasmodium falciparum infections to artemisinin-based combination treatments on the Kenyan coast. PLoS One 6 , e26005 doi:10.1371/journal.pone.0026005 (2011).
10. Boyle, M. J., Richards, J. S., Gilson, P. R., Chai, W. \& Beeson, J. G. Interactions with heparin-like molecules during erythrocyte invasion by Plasmodium falciparum merozoites. Blood 115, 4559-4568 (2010).

11. Clark, D. L., Su, S. \& Davidson, E. A. Saccharide anions as inhibitors of the malaria parasite. Glycoconj. J. 14, 473-479 (1997).

12. Evans, S. G., Morrison, D., Kaneko, Y. \& Havlik, I. The effect of curdlan sulphate on development in vitro of Plasmodium falciparum. Trans. R. Soc. Trop. Med. Hyg. 92, 87-89 (1998).

13. Butcher, G. A., Parish, C. R. \& Cowden, W. B. Inhibition of growth in vitro of Plasmodium falciparum by complex polysaccharides. Trans. R. Soc. Trop. Med. Hyg. 82, 558-559 (1988).

14. Xiao, L., Yang, C., Patterson, P. S., Udhayakumar, V. \& Lal, A. A. Sulfated polyanions inhibit invasion of erythrocytes by plasmodial merozoites and cytoadherence of endothelial cells to parasitized erythrocytes. Infect. Immun. 64, 1373-1378 (1996).

15. Adams, Y., Smith, S. L., Schwartz-Albiez, R. \& Andrews, K. T. Carrageenans inhibit the in vitro growth of Plasmodium falciparum and cytoadhesion to CD36. Parasitol. Res. 97, 290-294 doi:10.1007/s00436-005-1426-3 (2005).

16. Beuria, M. K. \& Das, M. K. Dextran sulfate induced suppression of Plasmodium berghei parasitaemia. Indian J. Exp. Biol. 29, 284-285 (1991).

17. Chen, J. H., Lim, J. D., Sohn, E. H., Choi, Y. S. \& Han, E. T. Growth-inhibitory effect of a fucoidan from brown seaweed Undaria pinnatifida on Plasmodium parasites. Parasitol. Res. 104, 245-250 doi:10.1007/s00436-008-1182-2 (2009).

18. Kisilevsky, R. et al. Short-chain aliphatic polysulfonates inhibit the entry of Plasmodium into red blood cells. Antimicrob. Agents Chemother. 46, 2619-2626 (2002).

19. Crandall, I. E. et al. Sulfated cyclodextrins inhibit the entry of Plasmodium into red blood cells. Implications for malarial therapy. Biochem. Pharmacol. 73, 632-642 (2007).

20. Kobayashi, K. et al. Application of retrovirus-mediated expression cloning for receptor screening of a parasite. Anal. Biochem. 389, 80-82 (2009).

21. Kobayashi, K. et al. Plasmodium falciparum BAEBL binds to heparan sulfate proteoglycans on the human erythrocyte surface. J. Biol. Chem. 285, 1716-1725 (2010).

22. Maier, A. G. et al. Plasmodium falciparum erythrocyte invasion through glycophorin C and selection for Gerbich negativity in human populations. Nat. Med. 9, 87-92 (2003)

23. Baum, J. et al. Reticulocyte-binding protein homologue 5 - an essential adhesin involved in invasion of human erythrocytes by Plasmodium falciparum. Int. J. Parasitol. 39, 371-380 (2009).

24. Kulane, A. et al. Effect of different fractions of heparin on Plasmodium falciparum merozoite invasion of red blood cells in vitro. Am. J. Trop. Med. Hyg. 46, 589-594 (1992).

25. Barragan, A. et al. The duffy-binding-like domain 1 of Plasmodium falciparum erythrocyte membrane protein 1 (PfEMP1) is a heparan sulfate ligand that requires 12 mers for binding. Blood 95, 3594-3599 (2000).

26. Cao, J. et al. Rhoptry neck protein RON2 forms a complex with microneme protein AMA1 in Plasmodium falciparum merozoites. Parasitol Int 58, 29-35 (2009).

27. Collins, C. R., Withers-Martinez, C., Hackett, F. \& Blackman, M. J. An inhibitory antibody blocks interactions between components of the malarial invasion machinery. PLoS Pathog 5, e1000273 (2009).

28. Harris, K. S. et al. Binding hot spot for invasion inhibitory molecules on Plasmodium falciparum apical membrane antigen 1. Infect. Immun. 73, 6981-6989 (2005).

29. Golomb, M., Vora, A. C. \& Grandgenett, D. P. Purification of reverse transcriptase from avian retroviruses using affinity chromatography on heparin-sepharose. J. Virol. Methods 1, 157-165 (1980).

30. Rabenstein, D. L. Heparin and heparan sulfate: structure and function. Nat. Prod. Rep. 19, 312-331 (2002).

31. Treeck, M. et al. Functional analysis of the leading malaria vaccine candidate AMA-1 reveals an essential role for the cytoplasmic domain in the invasion process. PLoS Pathog 5, e1000322 doi:10.1371/journal.ppat.1000322 (2009).

32. Singh, S., Alam, M. M., Pal-Bhowmick, I., Brzostowski, J. A. \& Chitnis, C. E. Distinct external signals trigger sequential release of apical organelles during erythrocyte invasion by malaria parasites. PLoS Pathog 6, e1000746 (2010).

33. Stubbs, J. et al. Molecular mechanism for switching of $P$. falciparum invasion pathways into human erythrocytes. Science 309, 1384-1387 (2005).

34. Gaur, D. et al. Upregulation of expression of the reticulocyte homology gene 4 in the Plasmodium falciparum clone $\mathrm{Dd} 2$ is associated with a switch in the erythrocyte invasion pathway. Mol. Biochem. Parasitol. 145, 205-215 (2006).

35. Gilson, P. R. \& Crabb, B. S. Morphology and kinetics of the three distinct phases of red blood cell invasion by Plasmodium falciparum merozoites. Int. J. Parasitol. 39, 91-96 doi:10.1016/j.ijpara.2008.09.007 (2009).

36. Lew, V. L. \& Tiffert, T. Is invasion efficiency in malaria controlled by pre-invasion events?Trends Parasitol 23, 481-484 doi:10.1016/j.pt.2007.08.001 (2007).

37. Carruthers, V. B., Hakansson, S., Giddings, O. K. \& Sibley, L. D. Toxoplasma gondii uses sulfated proteoglycans for substrate and host cell attachment. Infect. Immun. 68, 4005-4011 (2000).

38. Radfar, A. et al. Synchronous culture of Plasmodium falciparum at high parasitemia levels. Nat Protoc 4, 1828-1844 (2009). 
39. Sim, B. K. et al. Primary structure of the 175 K Plasmodium falciparum erythrocyte binding antigen and identification of a peptide which elicits antibodies that inhibit malaria merozoite invasion. J. Cell Biol. 111, 1877-1884 (1990).

40. Narum, D. L. \& Thomas, A. W. Differential localization of full-length and processed forms of PF83/AMA-1 an apical membrane antigen of Plasmodium falciparum merozoites. Mol. Biochem. Parasitol. 67, 59-68 (1994).

41. Kennedy, M. C. et al. In vitro studies with recombinant Plasmodium falciparum apical membrane antigen 1 (AMA1): production and activity of an AMA1 vaccine and generation of a multiallelic response. Infect. Immun. 70, 6948-6960 (2002).

42. Mayer, D. C. et al. Polymorphism in the Plasmodium falciparum erythrocytebinding ligand JESEBL/EBA-181 alters its receptor specificity. Proc. Natl. Acad. Sci. U. S. A. 101, 2518-2523 (2004).

43. Gaur, D. et al. Recombinant Plasmodium falciparum reticulocyte homology protein 4 binds to erythrocytes and blocks invasion. Proc. Natl. Acad. Sci. U. S. A. 104, 17789-17794 (2007).

44. Kaneko, O. et al. Apical expression of three RhopH1/Clag proteins as components of the Plasmodium falciparum RhopH complex. Mol. Biochem. Parasitol. 143, 20-28 (2005)

45. Triglia, T., Tham, W. H., Hodder, A. \& Cowman, A. F. Reticulocyte binding protein homologues are key adhesins during erythrocyte invasion by Plasmodium falciparum. Cell Microbiol 11, 1671-1687 (2009).

46. Rayner, J. C., Galinski, M. R., Ingravallo, P. \& Barnwell, J. W. Two Plasmodium falciparum genes express merozoite proteins that are related to Plasmodium vivax and Plasmodium yoelii adhesive proteins involved in host cell selection and invasion. Proc. Natl. Acad. Sci. U. S. A. 97, 9648-9653 (2000).

47. Hayton, K. et al. Erythrocyte binding protein PfRH5 polymorphisms determine species-specific pathways of Plasmodium falciparum invasion. Cell Host Microbe 4, 40-51 (2008).

48. Kaneko, O., Soubes, S. C. \& Miller, L. H. Plasmodium falciparum: invasion of Aotus monkey red blood cells and adaptation to Aotus monkeys. Exp. Parasitol. 93, 116-119 (1999).

49. Kato, K., Sudo, A., Kobayashi, K., Tohya, Y. \& Akashi, H. Characterization of Plasmodium falciparum protein kinase 2. Mol. Biochem. Parasitol. 162, 87-95 (2008).

50. Kaneko, O., Fidock, D. A., Schwartz, O. M. \& Miller, L. H. Disruption of the Cterminal region of EBA-175 in the Dd2/Nm clone of Plasmodium falciparum does not affect erythrocyte invasion. Mol. Biochem. Parasitol. 110, 135-146 (2000).

51. Boyle, M. J. et al. Isolation of viable Plasmodium falciparum merozoites to define erythrocyte invasion events and advance vaccine and drug development. Proc. Natl. Acad. Sci. U. S. A. 107, 14378-14383 (2010).

52. Benting, J., Mattei, D. \& Lingelbach, K. Brefeldin A inhibits transport of the glycophorin-binding protein from Plasmodium falciparum into the host erythrocyte. Biochem. J. 300 (Pt 3), 821-826 (1994).
53. Hirano, H. \& Watanabe, T. Microsequencing of proteins electrotransferred onto immobilizing matrices from polyacrylamide gel electrophoresis: application to an insoluble protein. Electrophoresis 11, 573-580 (1990).

\section{Acknowledgments}

This study was supported by a JSPS Research Fellowship for Young Scientists, Grants-in-Aid for Young Scientists, and Scientific Research on Innovative Areas (3308) from the Ministry of Education, Culture, Science, Sports, and Technology (MEXT) and for Research on global health issues from the Ministry of Health, Labour and Welfare of Japan, Bio-oriented Technology Research Advancement Institution (BRAIN), The Naito Foundation, The Mochida Memorial Foundation for Medical and Pharmaceutical Research, and the Program to Disseminate Tenure Tracking System from the Japan Science and Technology Agency (JST). We thank Dr. Louis H. Miller for providing the antibodies against JESEBL and PfRH4; Dr. Osamu Kaneko for providing the antibody against clag3.1 and for instruction in metabolic labeling methods of parasite proteins; Drs. Carol A. Long and Kazutoyo Miura for providing the rabbit anti-AMA1-C1 antibody; and Dr. Takafumi Tsuboi for providing the rabbit antibody against PfRON2. We also thank MR4 for providing us with malaria parasites, contributed by Drs. Thomas E. Wellems, Arthur Talman, and Robert Sinden, and antibodies, contributed by Drs. David C. Kaslow and Alan Thomas.

\section{Author contributions}

K.Kobayashi and K.Kato designed the experiments. K.Kobayashi and R.T. carried out the experiments and analyzed the data. H.T., T.S., A.I., H.G., F.C.R., T.I., T.H. and H.A. contributed to the data analysis and discussion. K.Kobayashi and K.Kato wrote the manuscript. K. Kato supervised the study.

\section{Additional information}

Competing financial interests: The authors declare no competing financial interests.

How to cite this article: Kobayashi, K. et al. Analyses of Interactions Between Heparin and the Apical Surface Proteins of Plasmodium falciparum. Sci. Rep. 3, 3178; DOI:10.1038/ srep03178 (2013)

(i) $\Theta$ This work is licensed under a Creative Commons Attributionvisit http://creativecommons.org/licenses/by-nc-nd/3.0 


\section{SCIENTIFIC \\ REP RTS}

DOI: 10.1038/srep04349

SUBJECT AREAS:

PARASITE HOST

RESPONSE

PATHOGENS

PARASITE BIOLOGY

CLINICAL MICROBIOLOGY

\section{SCIENTIFIC REPORTS:}

$3: 3178$

DOI: $10.1038 /$ srep03178

(2013)

Published:

11 November 2013

Updated:

12 March 2014
A
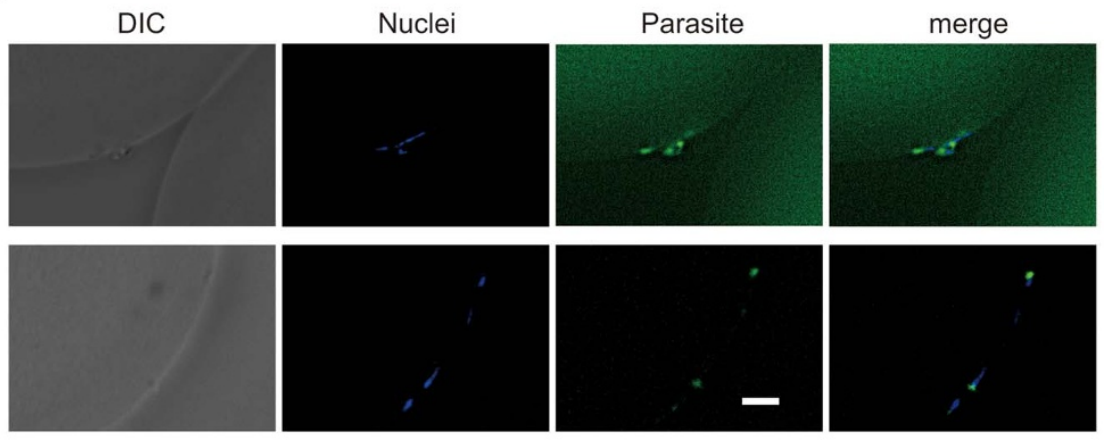

Figure 1 